\title{
Deep Q-network to produce polarization-independent perfect solar absorbers: a statistical report
}

\author{
Iman Sajedian ${ }^{1,2}$, Trevon Badloe ${ }^{2}$, Heon Lee ${ }^{1^{*}}$ and Junsuk Rho $2,3^{*}$ (I)
}

\begin{abstract}
Using reinforcement learning, a deep Q-network was used to design polarization-independent, perfect solar absorbers. The deep Q-network selected the geometrical properties and materials of a symmetric three-layer metamaterial made up of circular rods on top of two films. The combination of all the possible permutations gives around 500 billion possible designs. In around 30,000 steps, the deep Q-network was able to produce 1250 structures that have an integrated absorption of higher than $90 \%$ in the visible region, with a maximum of $97.6 \%$ and an integrated absorption of less than $10 \%$ in the 8-13 $\mu \mathrm{m}$ wavelength region, with a minimum of $1.37 \%$. A statistical analysis of the distribution of materials and geometrical parameters that make up the solar absorbers is presented.
\end{abstract}

Keywords: Reinforcement learning, Deep Q-learning, Perfect solar absorbers, Statistical analysis

\section{Introduction}

In the pursuit of renewable and green energy sources, the sun provides an enormous amount of energy waiting to be harvested in a meaningful way. Perfect solar absorbers play an important role in solar energy harvesting by converting photons into thermal energy $[1,2]$. Using perfect solar absorbers allows all of the absorbed energy to be used in the conversion process. An ideal solar energy absorber should have two main properties. First, it should absorb all wavelengths of electromagnetic radiation that reach the Earth, and second it should not radiate that absorbed energy away as heat. This process allows the absorbed solar energy to be completely converted to other forms of energy for practical everyday use. The proposed materials in this work can also be used as perfect absorbers in the visible region.

\footnotetext{
*Correspondence: heonlee@korea.ac.kr; jsrho@postech.ac.kr

${ }^{1}$ Department of Materials Science and Engineering, Korea University, Seoul 02842, Republic of Korea

${ }^{2}$ Department of Mechanical Engineering, Pohang University of Science and Technology (POSTECH), Pohang 37673, Republic of Korea

Full list of author information is available at the end of the article
}

One way to produce such solar absorbers is through metamaterials. Since their introduction by Pendry et al. [3], metamaterials have been used for numerous applications, such as light absorbers [4-15], cloaking devices $[16,17]$, and nonlinear optics $[18,19]$. By carefully designing the subwavelength geometrical properties of metamaterials, their optical properties can be manipulated for specific purposes. The design process is usually performed using knowledge from previous research and the intuition of the researcher. This can be an arduous process for more complex designs. Recently, artificial intelligence (AI) has been used to help find solutions to complex problems and uncover underlying relationships between the design parameter space and the optical properties in the field of nanophotonics [20]. Neural networks have been used for research in optics recently to design nanophotonic structures [21-27] and chiral metamaterials [28], predict the optical properties of structures [29], and for signal processing [30].

The deep Q-network (DQN), a reinforcement learning algorithm is a powerful tool that can be used to optimize solutions for a problem [29, 31-35] by acting as an intelligent search. Through exploration, the network takes 
actions and receives feedback, allowing it to learn about the parameter space and make intelligent choices. This method and its benchmarks have been explained in more detail in a number of articles [32, 33, 36, 37]. In contrast to other deep learning, reinforcement learning does not learn the hidden nonlinear relationships in a predetermined dataset but uses rewards and punishments in order to maximize a given reward. To start with there is no dataset, but through exploration and exploitation of the data space by an agent, it learns how to traverse the space and make good decisions to maximize its longterm reward.

\section{Methods}

\subsection{Structure}

A schematic of the initial structure is shown in Fig. 1a. It is composed of an array of nanocylinders on top of a silver back reflector and 2 film layers, all on a glass substrate. This structure was inspired by our previous experimental experience. The starting point can be chosen arbitrarily, but a well-educated guess can help to reach final results faster. The cylinders assure that the final structure will be polarization independent, while the bottom layer is a $200 \mathrm{~nm}$ silver back reflector, as is common in the design of many perfect absorbers. Lots of research has been published based on this type of structure with a variety of different number of layers and shapes $[6,38$, 39]. Here, the geometrical parameters and materials of the nanocylinders and layers are chosen by the DQN.

\subsection{Deep Q-network (DQN)}

The DQN was originally introduced as an AI agent that can play videogames at a level that can rival human players $[33,40]$. The DQN has been able to complete different games with the same algorithm. In videogames, each new screen is a new state where the agent can take an action, since there are so many possible states and actions, it is impossible to explore them all, or to use conventional algorithms to solve the game. A DQN starts by exploring a game and gradually learning the mechanics of it, the more the agent plays this game, the more it learns and is able to achieve higher scores. In this work, the DQN will learn the connection between the change of geometrical properties and their effect on final results through full wave FDTD simulations, and then use that knowledge to design structures that produce the optical responses that we desire. First, the environment is set up, this includes the initial structure design and the simulation environment, second, the actions that the agent can take to change the structure are decided and finally, the reward system is defined. The DQN algorithm that connects all these parts together is shown in Fig. 1b.

The decision of which action to take in a given state is decided by a neural network that is updated based on what it has learned. To improve the performance of the DQN, an auxiliary model is used alongside. This network is used to select the action for the agent to take, while the main DQN network is used to predict the Q-value of the state-action pair. This prevents the overestimation that is a problem in general DQN. At each iteration, two models are trained, and the weights of the a Starting structure

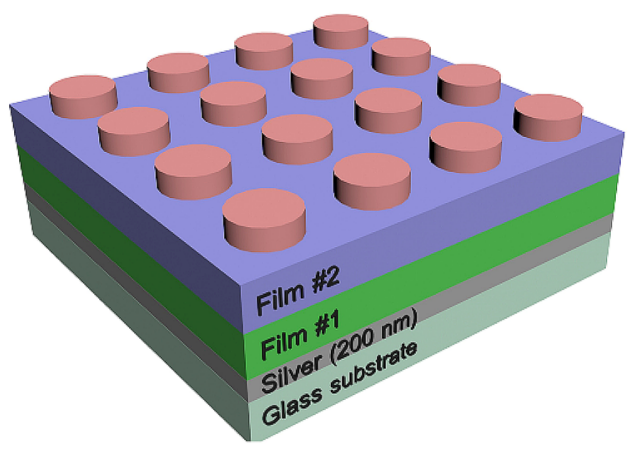

\section{b DDQN Model}

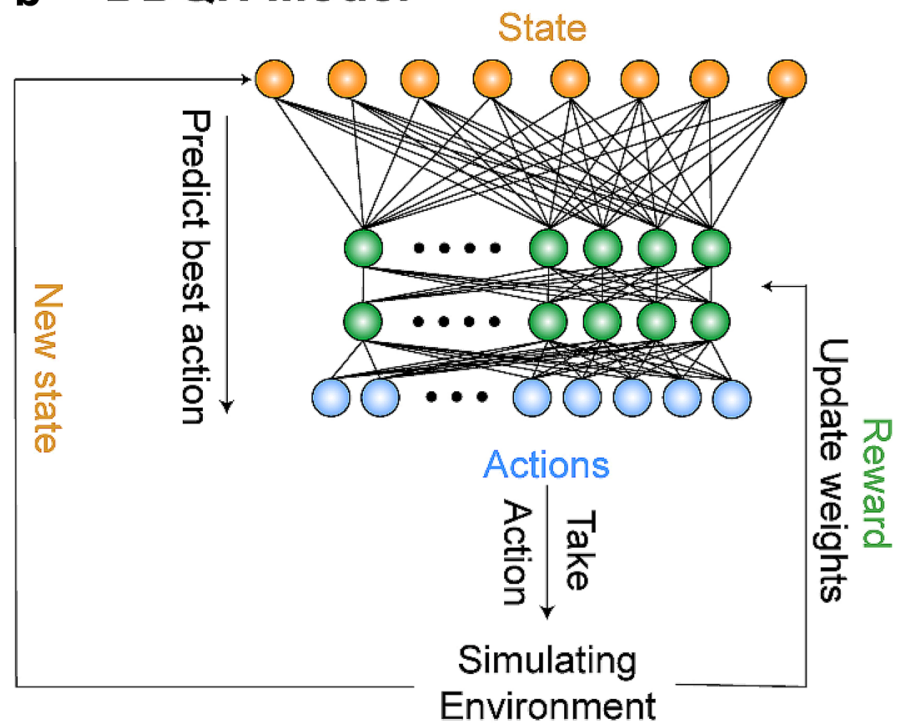

Fig. 1 a A schematic of the structure for the DDQN model to optimize and $\mathbf{b}$ the algorithm flowchart of the DDQN model 
target model are gained from the combination of the main model weights and the target model weights. This method helped the overestimation caused by using just one model. The auxiliary network is updated periodically with the parameters of the DQN. Since there are two networks working together, this is known as a double deep Q-network (DDQN) [41]. The rule for how an action is chosen is called the policy and the set of action, state, and policy form a Markov decision process (MDP). An MDP means that in a given state, the policy that is used to decide which action to take is based on the previous rewards gained from previous states and actions. The full details of this model and a pictorial comparison of the two q-network models is given in our previous work $[24,42]$. Each neural network has 3 hidden layers with 12 neurons.

\subsection{State}

The state, which is an array of the materials and geometrical properties of the structure, its variations, and limits are defined as follows:

- Cylinder material: 1 of 13 materials (Table 1).

- Film \#1 material: 1 of 13 materials (Table 1).

- Film \#2 material: 1 of 13 materials (Table 1).

- Cylinder diameter: 0-200 nm, step size: $10 \mathrm{~nm}$.

- Cylinder thickness: 0-200 nm, step size: $10 \mathrm{~nm}$.

- Film \#1 thickness: 0-2000 nm, step size: $10 \mathrm{~nm}$.

- Film \#2 thickness: 0-2000 nm, step size: $10 \mathrm{~nm}$.

- Gap between cylinders: 50-200 nm, step size: $10 \mathrm{~nm}$.

The total number of possible states is therefore, $13 \times$ $13 \times 13 \times 20 \times 20 \times 200 \times 200 \times 15=527,280,000,000$. Manually searching all of these states is impossible, but the DDQN can produce desirable results in a reasonable time. This will be discussed in more detail in the results section. It should be noted that the number of materials and geometrical properties can be chosen arbitrarily.

Table 1 The materials available to be used for the films and nanocylinders

\begin{tabular}{llcl}
\hline ID\# & Material & ID\# & Material \\
\hline 1 & $\mathrm{ZnS}$ & 8 & $\ln \mathrm{As}$ \\
2 & $\mathrm{TiO}_{2}$ & 9 & $\operatorname{InP}$ \\
3 & $\mathrm{PDMS}$ & 10 & $\mathrm{Ge}$ \\
4 & $\mathrm{Al}_{2} \mathrm{O}_{3}$ & 11 & $\mathrm{Si}$ \\
5 & $\mathrm{ZnO}$ & 12 & $\mathrm{Si}_{3} \mathrm{~N}_{4}$ \\
6 & $\mathrm{TiN}$ & 13 & $\mathrm{SiO}_{2}$ \\
7 & $\mathrm{GaAs}$ & & \\
\hline
\end{tabular}

Choosing a larger range of values could lead to better results but would take longer to train and converge. This is limited only by the available resources.

The initial state is defined as the central value of each parameter, i.e. cylinder, film \#1 and film \#2 materials: material 7 (GaAs), cylinder diameter: $100 \mathrm{~nm}$, cylinder thickness: $100 \mathrm{~nm}$, film \#1 and film \#2 thicknesses: $1000 \mathrm{~nm}$ and the spacing between cylinders: $100 \mathrm{~nm}$

\subsection{Actions}

The actions available to the agent to change the geometrical properties of the design at each step are shown in Table 2. As with all numerical methods, the parameter space is continuous, so it is discretized into smaller steps, as defined in "State" section. A step size of $10 \mathrm{~nm}$ for the cylinder diameter is chosen as it was deemed an appropriate accuracy through testing. At each update, the model learns from its previous states, actions and rewards and decides the best action to take next.

\subsection{Reward system}

The reward system gives feedback to the agent by giving information about how well it is learning. This is where the problem is set up to find a perfect solar absorber. A perfect solar absorber should have perfect absorption in the visible regime ( 350 to $800 \mathrm{~nm}$ ) to absorb all the solar energy, while having minimum absorption in the mid-IR range of $8 \mu \mathrm{m}$ to $13 \mu \mathrm{m}$ to not radiate it back out as heat. An area under the curve (AUC) value for each absorption spectrum was calculated in each region and the reward function was designed as follows:

Table 2 Definitions of the actions available to the agent

\begin{tabular}{ll}
\hline Action no. & Action definition \\
\hline 0 & Decrease the spacing between cylinders by $10 \mathrm{~nm}$. \\
1 & Increase the spacing between cylinders by $10 \mathrm{~nm}$. \\
2 & Decrease the height of the cylinder by $10 \mathrm{~nm}$. \\
3 & Increase the height of the cylinder by $10 \mathrm{~nm}$. \\
4 & Decrease the diameter of the cylinders by $10 \mathrm{~nm}$. \\
5 & Increase the diameter of the cylinders by $10 \mathrm{~nm}$. \\
6 & Decrease the thickness of film \#1 by $10 \mathrm{~nm}$. \\
8 & Increase the thickness of film \#1 by $10 \mathrm{~nm}$. \\
9 & Decrease the thickness of film \#2 by $10 \mathrm{~nm}$. \\
10 & Increase the thickness of film \#2 by $10 \mathrm{~nm}$. \\
11 & Decrease the material ID of cylinders by 1. \\
12 & Increase the material ID of cylinders by 1. \\
13 & Decrease the material ID of the film \#1 by 1. \\
14 & Increase the material ID of the film \#1 by 1. \\
15 & Decrease the material ID of the film \#2 by 1. \\
& Increase the material ID of the film \#2 by 1. \\
\hline
\end{tabular}




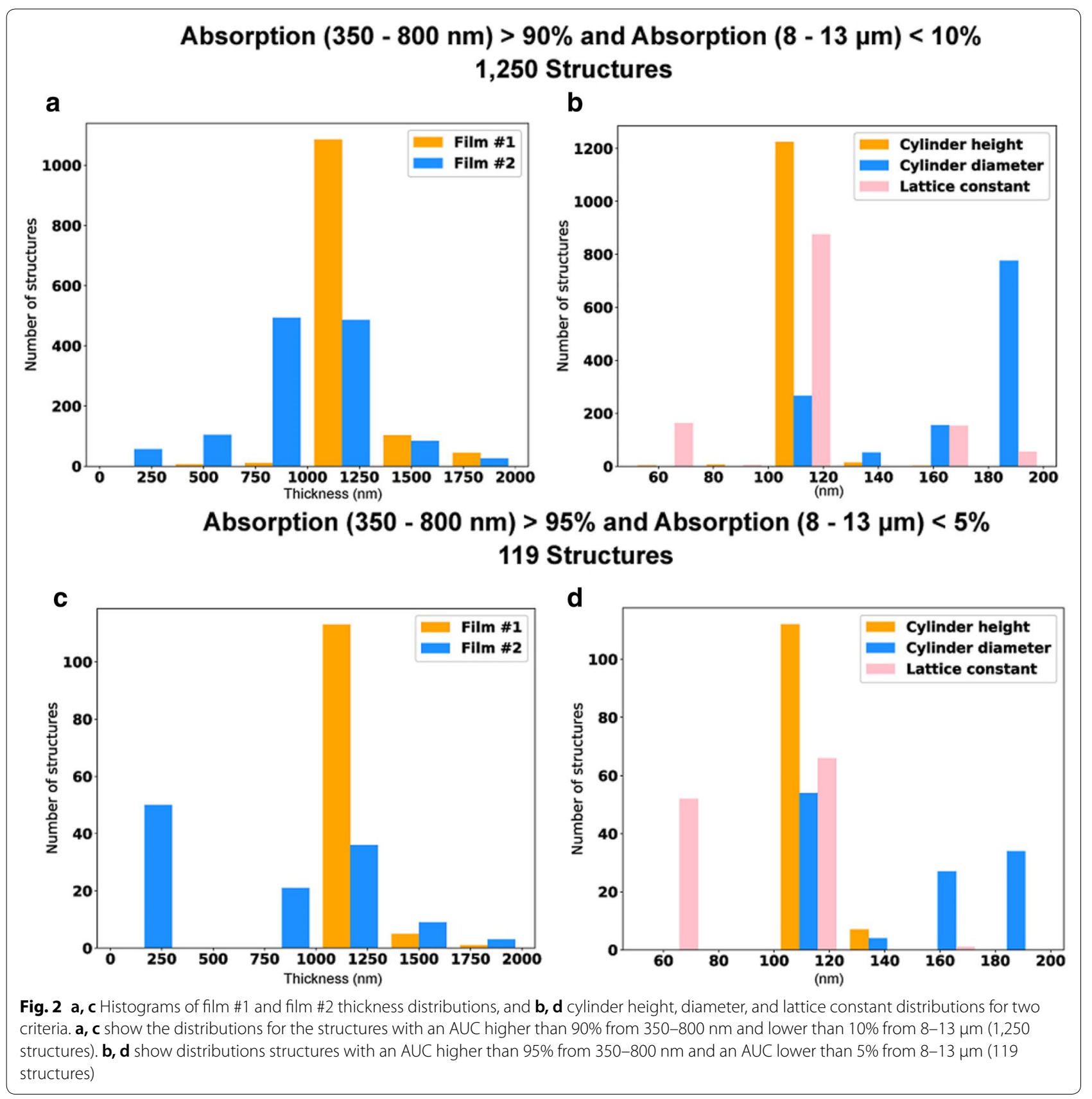

reward $=200+$ absorptionAUC $(350-800 \mathrm{~nm}) \%$

$$
\text { - absorptionAUC }(8-13 \mu m) \%
$$

200 is added to the reward to make sure that it remains positive. An ideal structure will gain a reward of 300, while the worst structure gets a reward of 100 (since the AUC ranges from 0 to $100 \%$ ). The absorption over each range of wavelengths was calculated with power monitors for reflection $(\mathrm{R})$ and transmission $(\mathrm{T})$, taking the absorption (A) to be $\mathrm{A}=1-\mathrm{R}-\mathrm{T}$.

\section{Results and discussion}

The simulations were performed using a computer with a $3.40 \mathrm{GHz}$ 16-core processor, $64 \mathrm{~GB}$ of RAM, and an NVIDIA RTX 2070 GPU with 8 GB DDR6 RAM. At each step, the DDQN code was run in Python for the AI calculations and connected to Lumerical, a commercial FDTD solver, to evaluate its predictions. All material data was taken from the inbuild database. This method requires a PC with both a strong CPU for the FDTD simulations and GPU for the neural network calculations. With this 


\section{a Absorption (350-800 nm) $>90 \%$ Absorption (8 - $13 \mu \mathrm{m})<10 \%$ 1,250 Structures}

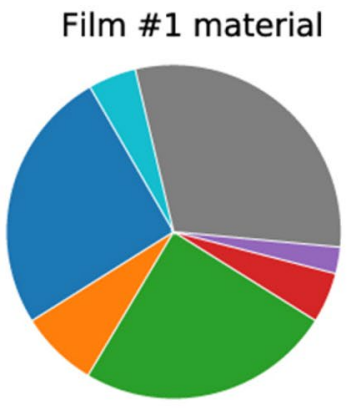

Film \#2 material

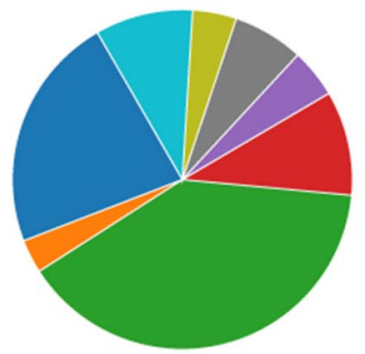

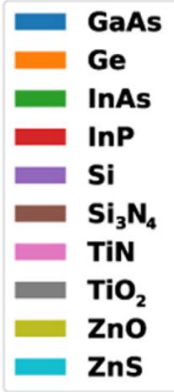

Cylinder material

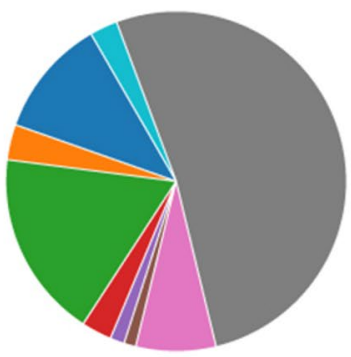

b Absorption (350-800 nm) $>95 \%$ Absorption (8 - $13 \mu \mathrm{m})<5 \%$ 119 Structures

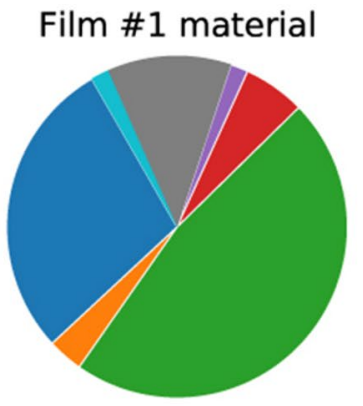

Film \#2 material

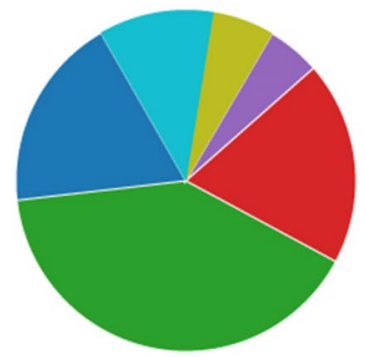

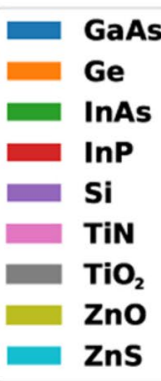

Cylinder material

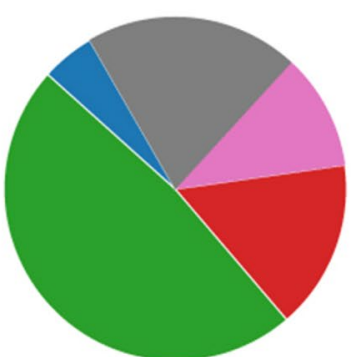

Fig. 3 Pie charts of (a) the material distributions of film \#1, film\# 2 and the cylinder for structures with an AUC higher than $90 \%$ from 350 to $800 \mathrm{~nm}$ and an AUC lower than 10\% from 8 to $13 \mu \mathrm{m}$ (1250 structures) and (b) for the structures with an AUC higher than $95 \%$ from 350 to $800 \mathrm{~nm}$ and an AUC lower than $5 \%$ from 8 to $13 \mu \mathrm{m}$ (119 structures)

setup it took around 1 month, where around 35,000 steps were taken. In the process of uncovering the best structures, the DDQN finds many similar solutions with small differences in their rewards. This means that many structures with acceptable results and different geometrical properties are discovered, allowing for a statistical analysis. A situation called backflipping occurred, which means that the model is stuck in a single configuration. This issue was fixed by tuning the hyperparameters. Figure 2 shows histograms of the distributions of different geometrical properties for film \#1, film \#2, the cylinder and the lattice constant. These graphs are prepared for two different categories. The first shows the top $10 \%$ of structures, which have an AUC of higher than $90 \%$ in the

Table 3 Some of highest efficiency structures by the DDQN

\begin{tabular}{|c|c|c|c|c|c|c|c|c|c|}
\hline Film \#1 mat. & Film \#2 mat. & $\begin{array}{l}\text { Film \#1 } \\
\text { height } \\
(\mathrm{nm})\end{array}$ & $\begin{array}{l}\text { Film \#2 } \\
\text { height } \\
(\mathrm{nm})\end{array}$ & Cylinder mat. & $\begin{array}{l}\text { Cylinder } \\
\text { height } \\
(\mathrm{nm})\end{array}$ & $\begin{array}{l}\text { Cylinder } \\
\text { diameter } \\
(\mathrm{nm})\end{array}$ & $\begin{array}{l}\text { Lattice } \\
\text { spacing } \\
(\mathrm{nm})\end{array}$ & $\begin{array}{l}\text { Abs. AUC (350 } \\
-800 \mathrm{~nm}) \%\end{array}$ & $\begin{array}{l}\text { Abs. AUC (8 } \\
-13 \mu \mathrm{m}) \%\end{array}$ \\
\hline Ge & GaAs & 1120 & 40 & InAs & 100 & 100 & 50 & 97.61 & 1.37 \\
\hline InAs & $\ln P$ & 1000 & 240 & InAs & 100 & 100 & 50 & 97.32 & 1.50 \\
\hline GaAs & $\ln P$ & 1120 & 40 & InAs & 100 & 100 & 50 & 97.05 & 2.48 \\
\hline InAs & GaAs & 1120 & 40 & InAs & 100 & 100 & 50 & 96.75 & 2.56 \\
\hline InAs & GaAs & 1110 & 40 & InAs & 100 & 100 & 50 & 96.74 & 2.67 \\
\hline GaAs & GaAs & 1120 & 40 & InAs & 100 & 100 & 50 & 96.57 & 2.58 \\
\hline InAs & $\mathrm{ZnO}$ & 1300 & 290 & TiN & 140 & 100 & 120 & 96.54 & 2.83 \\
\hline $\ln A s$ & $\ln A s$ & 1000 & 160 & $\ln A s$ & 100 & 100 & 50 & 96.53 & 2.85 \\
\hline
\end{tabular}



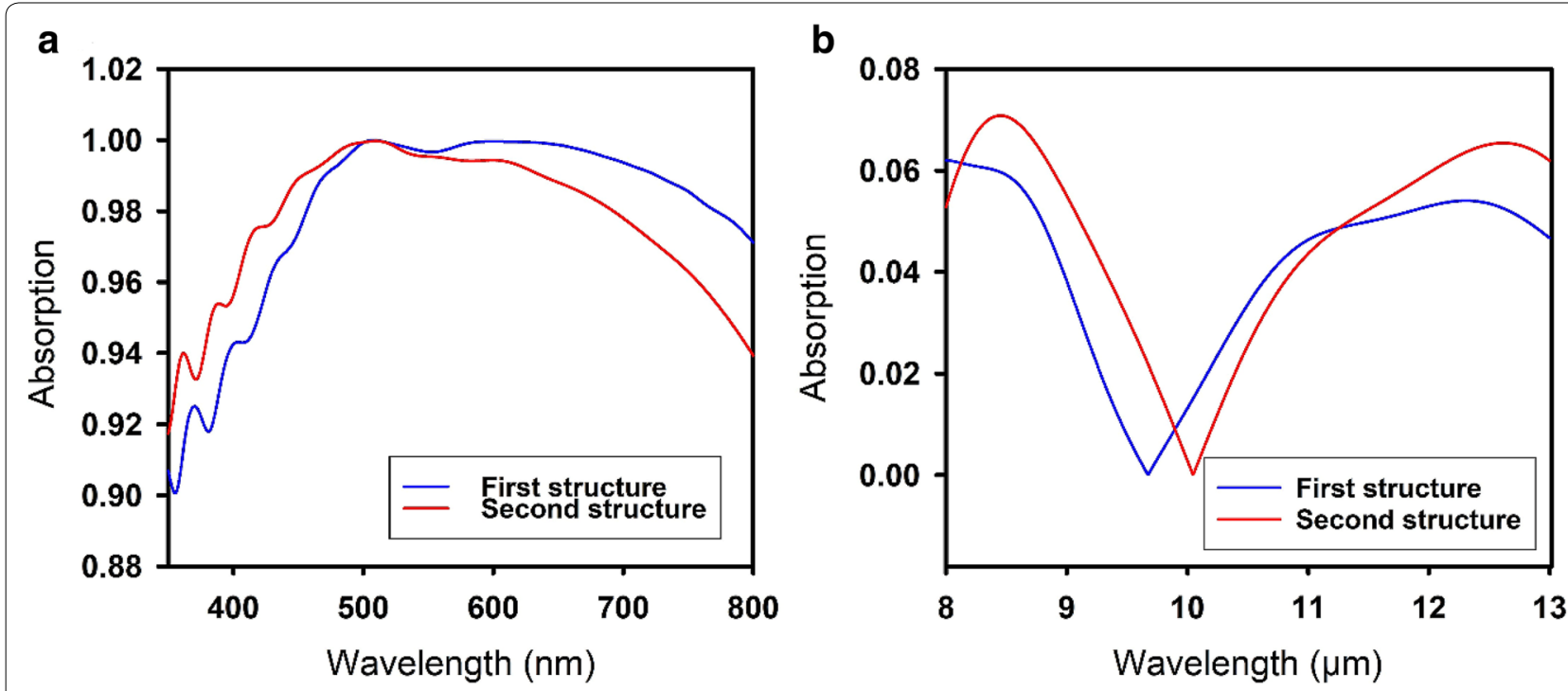

Fig. 4 Absorption curves of the top two performing structures from Table 3 for (a) $350-800 \mathrm{~nm}$ and (b) $8-13 \mu \mathrm{m}$

350 to $800 \mathrm{~nm}$ wavelength region and lower than $10 \%$ in the $8 \mu \mathrm{m}$ to $13 \mu \mathrm{m}$ region. The second displays the top $5 \%$ of structures, which have an AUC higher than $95 \%$ in the 350 to $800 \mathrm{~nm}$ wavelength region and lower than $5 \%$ in the $8 \mu \mathrm{m}$ to $13 \mu \mathrm{m}$ region. The DDQN produced 119 structures with these properties. The DDQN uncovered 1250 structures with these properties. shows histograms of the distributions of different geometrical properties for film \#1, film \#2, the cylinder and the lattice constant. These graphs are prepared for two different categories. The first shows the top $10 \%$ of structures, which have an AUC of higher than $90 \%$ in the 350 to $800 \mathrm{~nm}$ wavelength region and lower than $10 \%$ in the $8 \mu \mathrm{m}$ to $13 \mu \mathrm{m}$ region. The second displays the top $5 \%$ of structures, which have an AUC higher than $95 \%$ in the 350 to $800 \mathrm{~nm}$ wavelength region and lower than $5 \%$ in the $8 \mu \mathrm{m}$ to $13 \mu \mathrm{m}$ region. The DDQN produced 119 structures with these properties. The DDQN uncovered 1250 structures with these properties.

The distributions of the material choices for film \#1, film \#2 and the cylinder are displayed in the pie charts in Fig. 3 for the same categories as previously described. These plots reflect which materials should be chosen to obtain perfect solar absorbers. Table 3 shows the materials and geometric parameters of the top 8 performing structures discovered by the DDQN, with the absorption curves of the top 2 shown in Fig. 4. In comparison with human findings, A. Al-Rjoub et al. [43] reported almost identical theoretical and experimental results of a $95.2 \%$ and $9.8 \%$ absorption in the first and second wavelength regions.

\section{Conclusions}

A DDQN was used to design structures to be used as solar perfect absorbers in a parameter space that allows for 527 billion possible designs. Using a variety of materials, it was able to produce around 1250 perfect solar absorbers in around 35,000 steps. Each structure has an AUC higher than $90 \%$ in the visible region with low absorption in the $8 \mu \mathrm{m}$ to $13 \mu \mathrm{m}$ region. A statistical analysis was produced to help readers choose suitable geometrical properties and materials based on their fabrication limitations to design a perfect solar absorber.

\section{Acknowledgements}

Not applicable.

\section{Authors' contributions}

JR and IS conceived the idea and initiated the project. IS performed the simulations and analyzed the data. IS and TB wrote the manuscript and created the figures. All authors participated in the discussion and approved the final manuscript. JR and HL guided the entire work. All authors read and approved the final manuscript.

\section{Funding}

This work was financially supported by the National Research Foundation (NRF) grants (NRF-2019R1A2C3003129, CAMM-2019M3A6B3030637, NRF2019R1A5A8080290, NRF-2018M3D1A1058997) funded by the Ministry of Science and ICT (MSIT), Republic of Korea.

\section{Availability of data and materials}

The datasets generated and/or analyzed during the current study are not publicly available due to the funding agency's regulations but are available from the corresponding author on reasonable request.

\section{Competing interests}

The authors declare no competing interests.

\section{Author details}

${ }^{1}$ Department of Materials Science and Engineering, Korea University, Seoul 02842, Republic of Korea. ${ }^{2}$ Department of Mechanical Engineering, 
Pohang University of Science and Technology (POSTECH), Pohang 37673 Republic of Korea. ${ }^{3}$ Department of Chemical Engineering, Pohang University of Science and Technology (POSTECH), Pohang 37673, Republic of Korea.

Received: 7 May 2020 Accepted: 23 June 2020

Published online: 03 August 2020

\section{References}

1. Y. Li, D. Li, D. Zhou, C. Chi, S. Yang, B. Huang, Sol. RRL 2, 1800057 (2018)

2. Z. Zhou, E. Sakr, Y. Sun, P. Bermel, Nanophotonics 5, 1 (2016)

3. D.R. Smith, Science 305, 788 (2004)

4. J. Cong, Z. Zhou, B. Yun, L. Lv, H. Yao, Y. Fu, N. Ren, Opt. Lett. 41, 1965 (2016)

5. X. Tian, Z.-Y. Li, Photonics Res. 4, 146 (2016)

6. P. Yu, L.V. Besteiro, Y. Huang, J. Wu, L. Fu, H.H. Tan, C. Jagadish, G.P. Wiederrecht, A.O. Govorov, Z. Wang, Adv. Opt. Mater. 7, 1800995 (2019)

7. T. Badloe, I. Kim, J. Rho, Sci. Rep. 10, 4522 (2020)

8. T. Badloe, J. Mun, J. Rho, J. Nanomater. 2017, 1 (2017)

9. I. Kim, S. So, A.S. Rana, M.Q. Mehmood, J. Rho, Nanophotonics 7, 1827 (2018)

10. D. Lee, S.Y. Han, Y. Jeong, D.M. Nguyen, G. Yoon, J. Mun, J. Chae, J.H. Lee, J.G. Ok, G.Y. Jung, H.J. Park, K. Kim, J. Rho, Sci. Rep. 8, 12393 (2018)

11. D.M. Nguyen, D. Lee, J. Rho, Sci. Rep. 7, 2611 (2017)

12. N. Raeis-Hosseini, J. Rho, Appl. Sci. 9, 564 (2019)

13. A.S. Rana, M.Q. Mehmood, H. Jeong, I. Kim, J. Rho, Sci. Rep. 8, 2443 (2018)

14. G. Yoon, S. So, M. Kim, J. Mun, R. Ma, J. Rho, Nano. Converg. 4, 36 (2017)

15. N. Mahmood, I. Kim, M.Q. Mehmood, H. Jeong, A. Akbar, D. Lee, M. Saleem, M. Zubair, M.S. Anwar, F.A. Tahir, J. Rho, Nanoscale 10, 18323 (2018)

16. W. Cai, U.K. Chettiar, A.V. Kildishev, V.M. Shalaev, Nat. Photonics 1, 224 (2007)

17. M. Manjappa, P. Pitchappa, N. Wang, C. Lee, R. Singh, Adv. Opt. Mater. 6, 1800141 (2018)

18. I. Sajedian, A. Zakery, J. Rho, Opt. Commun. 397, 17 (2017)

19. I. Sajedian, I. Kim, A. Zakery, J. Rho, Opt. Commun. 401, 66 (2017)

20. S. So, T. Badloe, J. Noh, J. Bravo-Abad, J. Rho, Nanophotonics $\mathbf{9}, 1041$ (2020)

21. D. Liu, Y. Tan, E. Khoram, Z. Yu, ACS Photonics 5, 1365 (2018)

22. J. Peurifoy, Y. Shen, L. Jing, Y. Yang, F. Cano-Renteria, B.G. DeLacy, J.D. Joannopoulos, M. Tegmark, M. Solja, Sci. Adv. 4, EAAR4206 (2018)
23. J. Peurifoy, Y. Shen, Y. Yang, L. Jing, F. Cano-Renteria, J. Joannopoulos, M. Tegmark, M. Soljačić, in Frontiers in Optics 2017 (OSA, Washington, D.C., 2017), p. FTh4A.4

24. I. Sajedian, T. Badloe, J. Rho, Opt. Express 27, 5874 (2019)

25. I. Malkiel, M. Mrejen, A. Nagler, U. Arieli, L. Wolf, H. Suchowski, Light. Sci. Appl. 7, 60 (2018)

26. S. So, J. Mun, J. Rho, A.C.S. Appl, Mater. Interfaces 11, 24264 (2019)

27. S. So, J. Rho, Nanophotonics 8, 1255 (2019)

28. W. Ma, F. Cheng, Y. Liu, ACS. Nano. 12, 6326 (2018)

29. I. Sajedian, J. Kim, J. Rho, Microsyst. Nanoeng. 5, 27 (2019)

30. I. Sajedian, J. Rho, Nano. Converg. 6, 27 (2019)

31. T. Badloe, I. Kim, J. Rho, Phys. Chem. Chem. Phys. 22, 2337 (2020)

32. M. Bukov, A.G.R. Day, D. Sels, P. Weinberg, A. Polkovnikov, P. Mehta, Phys. Rev. X 8, 031086 (2018)

33. V. Mnih, K. Kavukcuoglu, D. Silver, A.A. Rusu, J. Veness, M.G. Bellemare, A. Graves, M. Riedmiller, A.K. Fidjeland, G. Ostrovski, S. Petersen, C. Beattie, A. Sadik, I. Antonoglou, H. King, D. Kumaran, D. Wierstra, S. Legg, D. Hassabis, Nature 518, 529 (2015)

34. I. Sajedian, H. Lee, J. Rho, Sci. Rep. 9, 10899 (2019)

35. I. Sajedian, H. Lee, J. Rho, Sol. Energy 195, 670 (2020)

36. T. Hester, M. Vecerik, O. Pietquin, M. Lanctot, T. Schaul, B. Piot, D. Horgan, J. Quan, A. Sendonaris, G. Dulac-Arnold, I. Osband, J. Agapiou, J. Z. Leibo, and A. Gruslys, ArXiv:1704.03732 (2017)

37. Q. Zhang, M. Lin, L.T. Yang, Z. Chen, S.U. Khan, P. Li, I.E.E.E. Trans, Serv. Comput. 12, 739 (2019)

38. A.K. Azad, W.J.M. Kort-Kamp, M. Sykora, N.R. Weisse-Bernstein, T.S. Luk, A.J. Taylor, D.A.R. Dalvit, H.-T. Chen, Sci. Rep. 6, 20347 (2016)

39. H. Wang, L. Wang, Opt. Express 21, A1078 (2013)

40. V. Mnih, K. Kavukcuoglu, D. Silver, A. Graves, I. Antonoglou, D. Wierstra, and M. Riedmiller, ArXiv:1312.5602 (2013)

41. H. van Hasselt, A. Guez, and D. Silver, ArXiv:1509.06461 (2015)

42. R. S. Sutton and A. G. Barto, Reinforcement Learning: An Introduction (The MIT Press, 1992)

43. A. AL-Rjoub, L. Rebouta, P. Costa, N.P. Barradas, E. Alves, P.J. Ferreira, K. Abderrafi, A. Matilainen, K. Pischow, Sol. Energy 172, 177 (2018)

\section{Publisher's Note}

Springer Nature remains neutral with regard to jurisdictional claims in published maps and institutional affiliations.

\section{Submit your manuscript to a SpringerOpen ${ }^{\odot}$ journal and benefit from:}

- Convenient online submission

- Rigorous peer review

- Open access: articles freely available online

- High visibility within the field

- Retaining the copyright to your article

Submit your next manuscript at $\boldsymbol{\nabla}$ springeropen.com 